Revista Médico Cientifica,
Vol. 34, Núm. 1:9-15, 2021
ISSN 2218-8266/ISSN-L 1608-3849

\title{
Criobiopsia como método diagnóstico de neumonía por Pneumocystis jirovecii
}

\author{
Cryobiopsy as a diagnostic method for Pneumocystis jirovecii pneumonia \\ Yurielis Ramos Rodríguez*; Elsa Rueda Borrero*
}

* Médico Residente de Médicina Interna. Complejo Hospitalario Dr. Arnulfo Arias Madrid. Panamá

\section{RESUMEN}

Expondremos el caso de un masculino de 57 años con VIH de reciente diagnóstico. Profesional de la salud con historia de un mes de pérdida de peso y síntomas respiratorios. Se realiza la criobiopsia para aislar Pneumocystis en corte histopatológico. La criobiopsia es un método, relativamente nuevo, que permite obtención de muestras de mayor tamaño, por lo que aumenta la sensibilidad diagnóstica a un 95-100\% en enfermedad pulmonar intersticial indeterminada.

\section{ABSTRACT}

This is the case of a 57-year-old male with recently diagnosed HIV, a health care worker with 1-month history of weight loss and respiratory symptoms. Cryobiopsy is performed to isolate Pneumocystis in a histopathological sample. Cryobiopsy is a relatively new method that allows the obtention of larger samples, thus increasing the diagnostic sensitivity to $95-100 \%$ in indeterminate interstitial lung disease.
Correspondencia: yuriramos2892@gmail.com Recibido: 02/06/20 Aceptado: 19/03/21

\section{PALABRAS CLAVE:}

VIH; Neumonía por Pneumocystis; Infecciones oportunistas.

KEY WORDS:

HIV; Pneumocystis pneumonia; opportunistic infections.

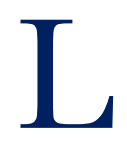

a infección por el virus de la inmunodeficiencia humana (VIH) es cada vez más prominente a pesar de la lucha contra el mismo. Las infecciones oportunistas tienen un lugar importante en el manejo de individuos seropositivos, como es la infección por Pneumocystis jirovecii, el agente causal de neumonía en estos pacientes. Su curso clínico es heterogéneo y para el diagnóstico se requiere la detección del hongo, ya sea por técnicas moleculares o visualización directa de su forma trófica o quística en secreciones respiratorias. Dicha muestra era previamente obtenida por inducción del esputo, lavado broncoalveolar o biopsia transbronquial. En ocasiones, se inicia la terapia sopesando el riesgo de la toxicidad que podría acarrear. 


\section{ENFERMEDAD ACTUAL}

Se trata de un masculino de 57 años, profesional de la salud, que acude con historia de 1 mes de tos seca, fiebre de predominio nocturno, pérdida de peso involuntaria de más de 20 libras y debilidad generalizada.

\section{ANTECEDENTES PERSONALES}

En ese momento, sin antecedentes personales patológicos, sin cirugías ni hospitalizaciones previas, sin hábitos de consumo tóxicos. Había mantenido relaciones sexuales con más de 10 mujeres en su vida, con uso irregular de métodos de barrera, actualmente unido. Niega sexo con hombres

\section{EXAMEN FÍSICO}

Inicialmente con presión arterial 98/64, frecuencia cardiaca 96 latidos por minuto, frecuencia respiratoria de 20 ciclos por minuto, oximetría de pulso $93 \%$ en aire ambiente.

Sin alteraciones neurológicas. Lesiones eritematosas descamativas en cuero cabelludo sugestivas de dermatitis atópica. Mucosa oral hidratada, sin lesiones. Tórax con correcta amplexión y amplexación sin tiraje, ruidos cardiacos regulares sin soplo. A la auscultación pulmonar se identifican crépitos finos en bases pulmonares. Abdomen blando, depresible, no doloroso. Sin edema en extremidades inferiores.

Se realiza una prueba rápida y otra confirmatoria de $\mathrm{VIH}$, ambas positivas.

Puesto que el paciente mostraba oximetría de pulso en $93 \%$, se toma gasometría arterial aire ambiente que reflejó alcalosis respiratoria y acidosis metabólica con marcada hipoxemia: pH 7.45, pCO2 $32 \mathrm{mmHg}$, pO2 $63 \mathrm{mmHg}$, Lactato $0.6 \mathrm{mmol} / \mathrm{L}$, HCO3-
$21.5 \mathrm{mmol} / \mathrm{L}$, SO2 93\% para un gradiente alvéoloarterial de $46.7 \mathrm{~mm} \mathrm{Hg}$, más elevado de lo esperado (18.25 mmHg, valor esperado).

\section{EXÁMENES DE GABINETE}

Se realiza radiografía posteroanterior de tórax que revela radiopacidad difusa en ambos campos pulmonares sugestivo de vidrio despulido. Ante los hallazgos, se realiza tomografía de tórax de alta resolución que evidencia patrón de vidrio despulido difuso en ambos pulmones con predominio biapical.

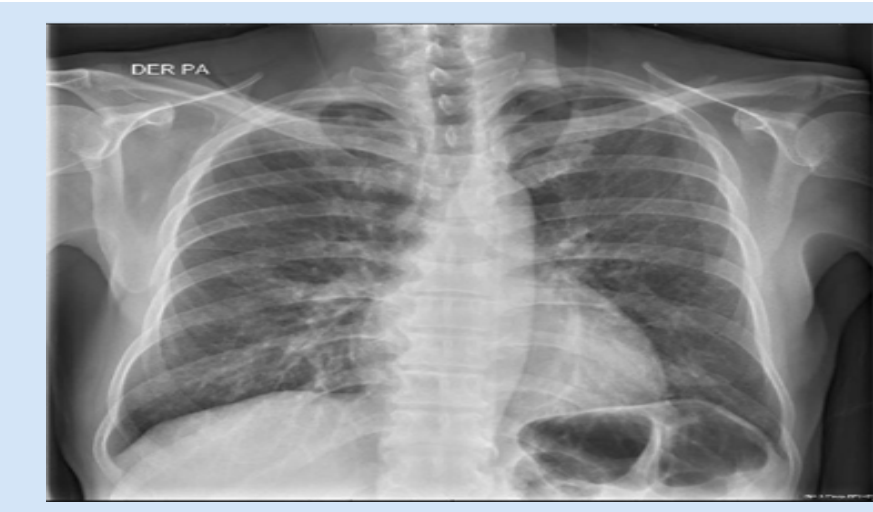

Figura 1. Radiografía de tórax con proyección posteroanterior

Fuente: Servicio de Radiología-Complejo Hospitalario Metropolitano Dr. Arnulfo Arias Madrid

\begin{tabular}{|ll|}
\hline \multicolumn{2}{|l|}{ Tabla 1: Laboratorios de ingreso } \\
\hline Hemoglobina & $13.4 \mathrm{~g} / \mathrm{dL}$ \\
Hematocrito & $38.50 \%$ \\
Plaquetas & $259 \mathrm{000} / \mathrm{\mu L}$ \\
Conteo de glóbulos blancos & $2900 / \mu \mathrm{L}$ \\
Neutrófilos & $47.50 \%$ \\
Glicemia & $122 \mathrm{mg} / \mathrm{dL}$ \\
Creatinina & $1.00 \mathrm{mg} / \mathrm{dL}$ \\
Nitrógeno de Urea & $15 \mathrm{mg} / \mathrm{dL}$ \\
Sodio & $133 \mathrm{mEq} / \mathrm{L}$ \\
Potasio & $4.3 \mathrm{mEq} / \mathrm{L}$ \\
Deshidrogenasa Láctica & $532 \mathrm{UI} / \mathrm{L}$ \\
Bilirrubina Total & $0.61 \mathrm{mg} / \mathrm{dL}$ \\
Bilirrubina Directa & $0.3 \mathrm{mg} / \mathrm{dL}$ \\
Aspartato aminotransferasa & $34 \mathrm{UI} / \mathrm{L}$ \\
Alanina aminotransferasa & $26 \mathrm{UI} / \mathrm{L}$ \\
\hline
\end{tabular}

Fuente: Laboratorio- Complejo Hospitalario Metropolitano Dr. Arnulfo Arias Madri 


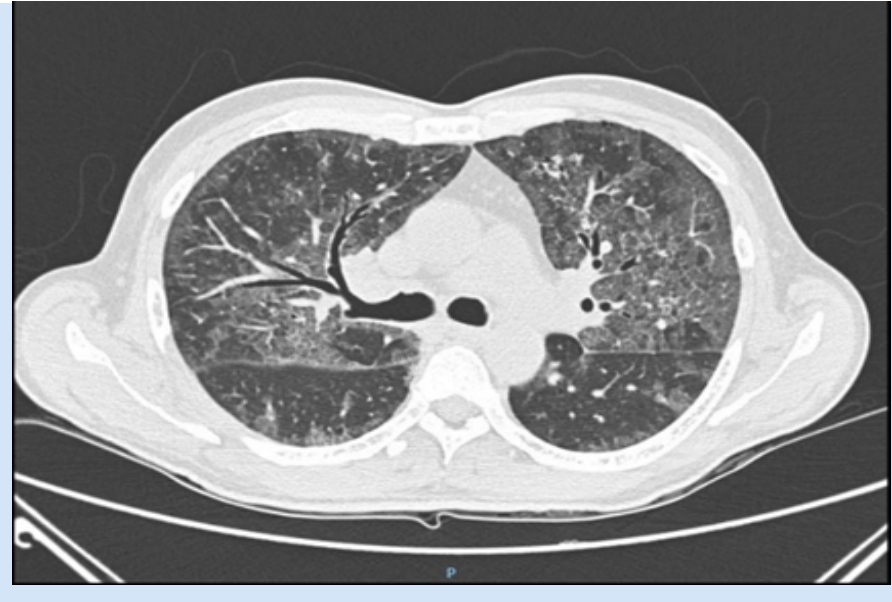

Figura 2. Corte axial de tomografía de tórax de alta resolución

Fuente: Servicio de Radiología-Complejo Hospitalario Metropolitano Dr. Arnulfo Arias Madrid

TRATAMIENTO

Además del diagnóstico inicial de $\mathrm{VIH}$, se sospecha de infección oportunista que, por las manifestaciones clínicas y hallazgos de laboratorio, sugería una infección de origen pulmonar. Se decide iniciar oxigenoterapia suplementaria y terapia empírica así:

- Cefotaxima 2 gramos intravenoso cada 8 horas y Azitromicina $500 \mathrm{mg}$ vía oral por 5 días para neumonía adquirida en la comunidad;

- Trimetoprima $16 \mathrm{mg} /$ Sulfametoxazol $80 \mathrm{mg}$ (TMP/SMX) 4 ampollas intravenosa tid para Pneumocystis jirovecii;

- De forma profiláctica, Itraconazol 200 mg vía oral cada día para Histoplasma capsulatum.

- Además, se prescribió de manera inicial prednisona $40 \mathrm{mg}$ vía oral por hallazgos de hipoxemia y gradiente $A$-a elevado.

- Se inició terapia antirretroviral con efavirenz + emtricitabina + tenofovir (Atripla).

\section{EVOLUCIÓN}

Se inician estrategias para aislamiento microbiológico en esputo, sin éxito, por lo que se consulta con servicio de neumología y se decide llevar a broncoscopia. Durante la misma no se evidencia lesión endobronquial; sin embargo, llamó la atención una escasa secreción desde ambos lóbulos superiores. Se realiza lavado endobronquial para estudios microbiológicos. Se decide realizar criobiopsia transbronquial de lóbulo superior derecho (RB1) y de lóbulo inferior izquierdo (RB6). Se envían muestras a microbiología y patología. El procedimiento involucró complicaciones inmediatas, en el control radiográfico se evidenció un pequeño neumotórax apical derecho de $<2 \mathrm{~cm}$ y se decide continuar oxígeno suplementario y vigilar en las siguientes horas. En las horas subsecuentes, el paciente desarrolla taquipnea, con ruidos pulmonares disminuidos en pulmón derecho; en el siguiente control radiográfico se evidencia aumento del neumotórax con requerimiento de colocación de tubo pleural que se retira 48 horas después sin complicaciones. Se recibe el PCR por Mycobacterium tuberculosis negativo y además obtenemos el resultado del $\mathrm{CD} 4+\mathrm{y}$ carga viral $(\mathrm{VIH}-1)$, los cuales reportaban 5 células $/ \mathrm{mm} 3$ y 340000 copias $/ \mathrm{ml}$, respectivamente. El paciente cursa con adecuada evolución clínica y se da egreso con seguimiento en consulta externa y prescripción de TMP $160 \mathrm{mg} / \mathrm{SMX}$ 800 mg 2 tabletas por vía oral cada 8 horas, decalaje de prednisona, dosis profiláctica de itraconazol para Histoplasmosis y terapia antirretroviral.

De manera ambulatoria se obtiene resultado de patología que reporta dos fragmentos de muestra histopatológica y en ambas se hallan características compatibles con infección por Pneumocystis jirovecii. El curso terapéutico de 21 días fue completado sin complicaciones y se continuó régimen profiláctico para dicho hongo con TMP 160mg/SMX 800mg 1 tableta vía oral cada día.

www.revistamedicocientifica.org 
A los seis meses, el paciente mejoró su estado general $y$ se encontró asintomático, con recuperación de su peso y con controles de CD4+ en 148 células $/ \mathrm{mm} 3$ y valores de carga viral $<20$ copias $/ \mathrm{ml}$.

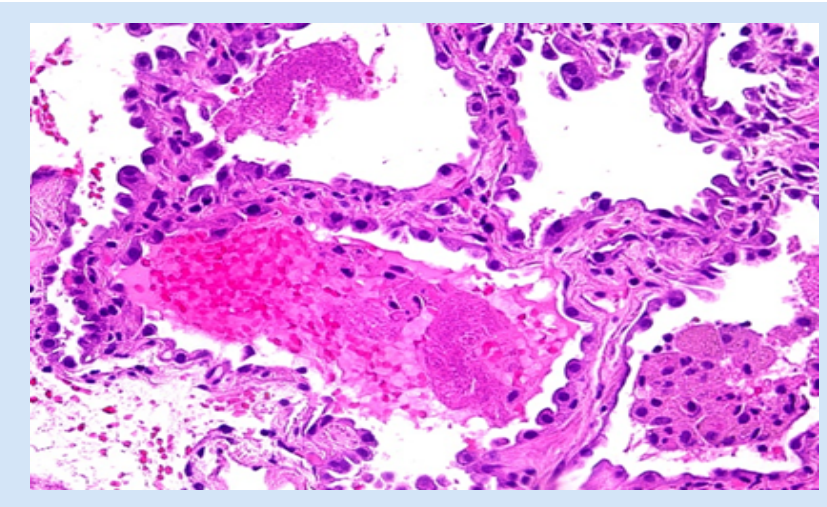

Figura 3. Exudado eosinofílico granular

Microscopía de luz. 40x. Tinción hematoxilina y eosina. La neumonía por Pneumocystis jirovecii muestra exudado eosinofílico granular llenando el lumen alveolar.

Fuente: Servicio de Patología-Complejo Hospitalario Metropolitano Dr. Arnulfo Arias Madrid

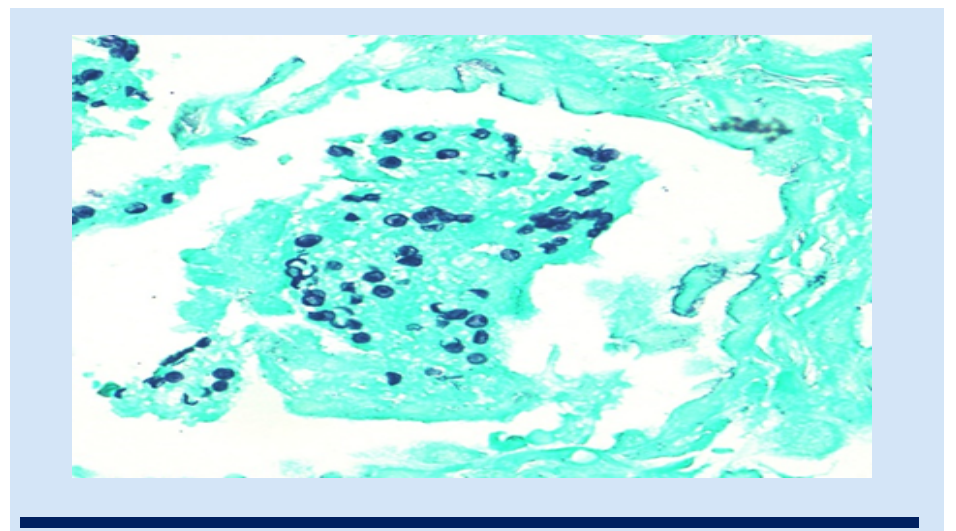

Figura 3. Exudados intraalveolares

Microscopía de luz. 40x. La tinción de Gomori destaca la presencia de Pneumocystis jirovecii dentro de los exudados intraalveolares. Los quistes son redondos a ovales con puntos oscuros colocados en el centro, pero también pueden mostrar formas colapsadas o en forma de media luna.

Fuente: Servicio de Patología-Complejo Hospitalario Metropolitano Dr. Arnulfo Arias Madrid

Rev Méd Cient. 2021;34(1):9-15

\section{DISCUSIÓN}

Panamá se posiciona a nivel regional como el segundo país con mayor cantidad de infectados con VIH en Centroamérica y el décimo en América Latina. ${ }^{[1]}$ Según datos de ONU SIDA publicados en el 2018, en nuestro país viven 26000 personas con VIH y aproximadamente 1300 adultos mayores de 15 años fueron nuevos diagnósticos, elevando la incidencia a 0.60 por cada 1000 habitantes. Asimismo, el documento reporta que $36 \%$ de los diagnósticos se realizan en fase SIDA con CD4 $+<200$ células/mm3, como el caso de nuestro paciente. ${ }^{[2]}$

Hoy por hoy, el diagnóstico de neumonía por Pneumocystis jirovecii (PCP, por sus siglas en inglés) sigue siendo difícil en nuestro medio, por lo que en muchas ocasiones se brinda tratamiento sin aislamiento del microorganismo; basándose esencialmente en probabilidad clínica, guías y estudios científicos que respaldan el inicio de dicho tratamiento.

Los primeros reportes alrededor de este microorganismo datan de 1909 por C. Chagas en pulmones de cuy. Inicialmente pensó que podría ser parecido al tripanosoma, pero 6 años más tarde Delanoe y Delanoe se encargaron de separarlas en una nueva especie: Pneumocystis, llamándolo así por su morfología quística y patogenia a nivel pulmonar. Por mucho tiempo se asoció a pacientes en estados de malnutrición y con enfermedades asociadas a estados de inmunodepresión hasta que apareció el síndrome de inmunodeficiencia adquirida (SIDA), donde su control solo se dio cuando apareció la terapia antirretroviral (TARV). ${ }^{[3]}$

Después de largo debate en el mundo taxonómico, se sabe que el Pneumocystis pertenece al reino de los hongos, pero cuenta con características atípicas ya que no crece in vitro en medios de cultivo, responde a agentes antiparasitarios como pentamidina $y$ cotrimoxazol y su pared celular contiene colesterol 
en vez de ergosterol, lo que explica su resistencia a la anfotericina B. Tiene dos formas de vida: la trófica (14 micras) y la quística (8-10 micras). La forma trófica se adhiere a las paredes alveolares y se nutre. La propia fibronectina y vitronectina presentes en el líquido alveolar ayudan a su unión y desencadena una serie de caminos hacia la activación de cinasas selectivas que contribuyen a la proliferación del Pneumocystis. Su eliminación depende de macrófagos alveolares y CD4+, los cuales están afectados en pacientes con VIH, inmunodeficiencias primarias, neoplasias sólidas y hematológicas. EI diagnóstico puede ser abordado en cuanto a imagen, microbiología e histopatología. ${ }^{[3]}$

En lo que concierne a imagen, los rayos $X$ de tórax pueden ser normales o tener datos inespecíficos. Se puede realizar una tomografía que es mucho más sensible y el hallazgo clásico se basa en infiltrados intersticiales difusos que podrían avanzar hasta consolidados, además de patrón de vidrio despulido; este último consistente con el hallazgo imagenológico en nuestro paciente. Otras cualidades no microbiológicas que contribuyen en el diagnóstico y que están presentes en nuestro caso son hipoxemia y elevación de la $\mathrm{LDH}>500 \mathrm{ml} / \mathrm{dL}^{\text {. }}{ }^{[4]}$

El tratamiento de elección descrito en las guías estadounidenses es Trimetoprima/Sulfametoxazol a dosis de TMP 15-20 mg y SMX 75-100 mg/kg/día vía intravenosa administrado cada 6 a 8 horas con la posibilidad de cambiar a vía oral cuando haya mejoría clínica del paciente. La terapia adyuvante con corticoides está indicada en PCP moderado a severo, es decir, que presente $\mathrm{PaO} 2<70 \mathrm{mmHg}$ aire ambiente o gradiente alvéolo-arterial $\geq 35 \mathrm{~mm} \mathrm{Hg}$. La administración del esteroide es en base a prednisona y se debe iniciar en las primeras 72 horas a 40mg vía oral bid por 5 días, luego 40mg vía oral qd del día 6 10 y finalmente $20 \mathrm{mg}$ vía oral qd hasta cumplir 21 días:[5] En el caso expuesto, brindamos la terapia estándar por el tiempo establecido de 3 semanas y además por criterios gasométricos, se clasificó la infección como severa e iniciamos terapia con corticoides.

Para el aislamiento microbiológico, se pueden adoptar varias estrategias y la dificultad radica en la obtención del esputo. ${ }^{[4]}$ En casos en que esto no se pueda, debido a que no hay producción de este, se recurre a su inducción. En casos como el de nuestro paciente, en que no haya muestra satisfactoria de esputo, se debe incurrir a estrategias más invasivas. Desde 1977 en la literatura mundial se han adoptado diferentes maneras de obtención de muestras y una de las primeras fue la punción transtorácica con aguja, utilizando los mismos agentes de tinción que en nuestros tiempos (Gomori, toluidina); sin embargo, evidenciaron una sensibilidad del $60 \%$ y altas tasas de complicaciones, hasta el $40 \%$ de los pacientes presentaron neumotórax. ${ }^{[6]}$

En la década de los ochenta, se introduce como técnica diagnóstica para neumonía por Pneumocystis la broncoscopia y el lavado broncoalveolar (BAL, por sus siglas en inglés). ${ }^{[7]} \mathrm{Al}$ estudiarse este método y todo aquel que depende de análisis histopatológico, se debe plantear la incertidumbre de los factores técnicos y de disposición de insumos, ya que la sensibilidad de la prueba depende de estas variables. La cantidad de muestra, la lectura adecuada del tejido y las tinciones aplicadas son cruciales. La literatura describe una fluctuación en la detección del hongo, ${ }^{[8]}$ en varios estudios la evidencia dictaba que el BAL es suficiente con sensibilidad mayor del 95\%; sin embargo, puede disminuir hasta un 50\% por lo anteriormente mencionado. Este procedimiento aunado a la biopsia transbronquial solidificaba el diagnóstico; no obstante, al pasar del tiempo y la tecnología, tomando en cuenta costos y complicaciones, se favorecía cada vez más solo realizar BAL para el diagnóstico de PCP, siempre y cuando se cuenten con insumos, equipo y personal capacitado. ${ }^{[7,9]}$ 
Aproximadamente 20 años atrás, se desarrolló una nueva estrategia mínimamente invasiva para la toma de muestras; al inicio fue específicamente diseñada para la toma de biopsia en enfermedades intersticiales pulmonares $y$, eventualmente, ha ganado terreno en otras aplicaciones. La criobiopsia es una tecnología en asiduo estudio y en cuyo inicio tenía altos porcentajes de complicaciones atribuibles, según algunos expertos, a la curva de aprendizaje. $^{[10]} \mathrm{El}$ procedimiento se basa en un agente congelante que puede ser dióxido de carbono y una sonda fría (cryoprobe) que se introduce por el broncoscopio. Tiene una punta de metal que se congela muy rápido y hay liberación de gas que ayuda a que se expanda y llegue a temperaturas de $79^{\circ} \mathrm{C}$; la temperatura óptima para tejido pulmonar es $-52^{\circ} \mathrm{C}$. El tejido se congela, se adhiere y se puede sacar dicho tejido para ser puesto en salina para despegarlo $y$, posteriormente, ser enviado a patología en formalina. ${ }^{[11]}$ La diferencia entre la criobiopsia y la biopsia transbronquial tradicional suele ser el tamaño obtenido de la muestra. La biopsia transbronquial obtiene muestras entre 1 a 3 milímetros y la criobiopsia desde 5 hasta 10 milímetros. A mayor tamaño mejores posibilidades de hallazgos histopatológicos. Además, involucra la disminución en gastos de salud y en recursos. En el caso de la biopsia transbronquial, se requiere una hospitalización de $48 \mathrm{~h}$ y la utilización de salón de operaciones con todo lo que involucra su uso. ${ }^{[12]} \mathrm{Se}$ ha demostrado que en pacientes VIH seropositivos, la criobiopsia transbronquial aunada al BAL tiene 93.3\% de rendimiento diagnóstico para patologías no intersticiales, entre ellas las de etiología infecciosa. ${ }^{[10]}$ Además, su aceptación y mejoría de la

\section{BIBLIOGRAFÍA}

[1] ONU SIDA. AIDSinfo Compare Data [Internet]. 2018. Disponible en:

http://aidsinfo.unaids.org/. práctica ha traído disminución en la tasa de complicaciones hasta un $4 \%$, volviendo esta técnica diagnóstica una opción conveniente. ${ }^{[13]}$

Todavía sigue siendo un campo en desarrollo, encontrando sus mayores dificultades en la esfera de su seguridad. Actualmente, las más grandes complicaciones todavía siguen siendo la hemorragia y el neumotórax. Esta técnica tiene el potencial de desarrollar neumotórax en el 33\% de los casos, de los cuales hasta el $6 \%$ requieren tubo pleural para la resolución del mismo. No hay manera de compararlo con la biopsia transbronquial, puesto que el neumotórax está dentro del protocolo quirúrgico. De igual manera, podrían desarrollar hemorragias moderadas a severas, que se han asociado a la toma de muestras de lóbulos inferiores de hasta $40 \%{ }^{[14,15]}$

\section{CONCLUSIÓN}

Todos estos esfuerzos globales para desarrollar técnicas cada vez más seguras y eficientes en el diagnóstico están orientadas a llegar a un diagnóstico basado en evidencia histológica y microbiológica, más que realizar tratamientos basados en sospechas y así cambiar el curso clínico de nuestros pacientes, al igual que utilizar de mejor forma los recursos en salud.

\section{AGRADECIMIENTOS}

Servicio de Neumología, especialmente a la Dra. Noriega, Dr. Hevia y MR Juan Pablo Byrne. A el Servicio de Patología y al Servicio de Infectología del Complejo Hospitalario Metropolitano Dr Arnulfo Arias Madrid.

[2] ONU SIDA. Country factsheets: PANAMA [Internet]. 2018. Disponible en:

https://www.unaids.org/es/regionscountries/countries/p anama.

[3] Catherinot E et al. Pneumocystis jirovecii pneumonia. Infect Dis Clin N Am 2010; 24:107-138 
[4] Lewis White P. Diagnosis and management of Pneumocystis jirovecii infection. Expert Review of Antiinfective therapy 2017;15(5):435-447.

[5] Panel on Guidelines for the Prevention and Treatment of Opportunistic Infections in Adults and Adolescents with HIV. Guidelines for the Prevention and Treatment of Opportunistic Infections in HIV-infected Adults and Adolescents: Recommendations from the Centers for Disease Control and Prevention, the National Institutes of Health, and the HIV Medicine Association of the Infectious Diseases Society of America. 2019. Disponible en: http://aidsinfo.nih.gov/contentfiles/lvguidelines/adult oi .pdf.

[6] Chaudhary $S$, et al. Percutaneous transthoracic needle aspiration of the lung. Am J Dis Child 1977; 131:902-7.

[7] Rodiño J, et al. Diagnóstico microscópico de neumonía por Pneumocystis jirovecii en muestras de lavado broncoalveolar y lavado orofaríngeo de pacientes inmunocomprometidos con neumonía. Biomedica 2011; 31:222-31.

[8] Griffiths $\mathrm{MH}$, et al. Diagnosis of pulmonary disease in human immunodeficiency virus infection: role of transbronchial biopsy and brochoalveolar lavage. Thorax 1989; 44:554-558

[9] Baughman R. Use of bronchoscopy in the diagnosis of infection in the immunocompromised host. Thorax 1994; 49:3-7

[10] Sánchez-Cabral O, et al. Utility of Transbronchial Lung Cryobiopsy in Non-Interstitial Diseases. Respiration 2017; 94:285-292.

[11] Barisione E. Competence in transbronchial cryobiopsy. Panminerva Med 2019; 61:290-7

[12] Hernández-González F et al. Utilidad de la criobiopsia en el diagnóstico de la enfermedad pulmonar intersticial difusa: análisis de rentabilidad y coste. Arch Bronconeumol. 2015;51(6):261-267.

[13] Chen A et al. Cryobiopsy: A Work in Progress. Ann Am ThoracSoc 2017;14(6):827-8.

[14] Tomasetti S, et al. Bronchoscopic Lung Cryobiopsy Increases Diagnostic Confidence in the Multidisciplinary Diagnosis of Idiopathic Pulmonary Fibrosis. Am J Respir Crit Care Med. 2016;193(7):745-752.

[15] Lodhi T, et al. Transbronchial Lung Cryobiopsy in Idiopathic Pulmonary Fibrosis: A State of the Art Review. Adv Ther. 2019; 36:2193-220. 